\title{
Researching Student Motivation
}

\author{
Sultan Ali R. Alkaabi, National Defence College, UAE \\ Warda Alkaabi, Ajman First Academy, UAE \\ Glen Vyver, Swinburne University Of Technology, Australia
}

\begin{abstract}
Motivation has been studied by different scientists in different fields of knowledge such as biology, psychology, and education for a long period, which has cultivated a wealth of knowledge in these disciplines. The richness in motivation theories poses complexity in motivation research. Due to these complexities, many researchers focus on using a single motivation theory in their studies. Using multiple motivation theories in a singular study is not a common practice in education research. This study presents a unifying framework for approaching motivation research in education which offers an opportunity to use multiple motivation theories in educational research, and other disciplines.
\end{abstract}

Keywords: Motivation; Psychology; Education, Student Motivation; Unifying Themes

\section{MOTIVATION: AN INTRODUCTION}

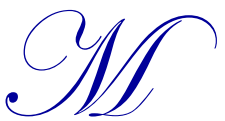

otivation is the force which provides the impetus for human behavior, causing individuals to initiate and sustain goal-directed actions (Jenkins \& Demaray, 2015). It is related to the person's will to embrace or get involved in a task or a process of action and serves to explain why individuals pursue some courses of action but avoid others (Schunk, Pintrich, \& Meece, 2008; Weiner, 1992). Motivation has been the focus of much research involving many theories and constructs (Wigfield, Eccles, Schiefele, Roeser, \& Davis-Kean, 2009).

Furthermore, motivation has been studied from multiple scientific perspectives including the cognitive, phenomenological, physiological and cultural dimensions (Ryan, 2012). Research into motivation crosses many disciplinary boundaries including psychology, education, and management. Research on motivation is vast in scope, spawning many theories. Furthermore, the discipline of psychology has spawned research into motivation and continues to be the epicentre of related research activity, and as a result, an examination of previous research via the prism of several of the major perspectives in psychology is important. This examination provides such an introduction with a strong conceptual foundation towards building a reference framework for student motivation research.

\section{Motivation Theories \& Perspectives}

The psychodynamic perspective focuses on biological factors and unconscious motivations. Freud argued that drives are the key motivators of human behavior, in particular sex and aggression, which includes control and mastery (McClelland, 1985). Drives, expressed directly and indirectly, build tension to achieve a state of satisfaction. The psychodynamic approach has evolved over time, and theorists now focus on motives and needs, including Freud's two basic drives but adding needs focused on self-esteem and relatedness to others (Mitchell, 1988; Shaver \& Mikulincer, 2005). Theorists also began to link motivation strongly to the term Affect, arguing that wishes and fears better expressed the forces that drive human behavior than the basic drives proposed by Freud (McClelland, 1985). Wishes and fears are both associated with arousal and emotional states. Whereas a wish represents the desired outcome, and becomes less intense after achieving that outcome, a fear represents an undesired outcome accompanied by unpleasant feelings (McClelland, 1985).

Experimental research has revealed that there is a strong unconscious element in motivation (Shaver \& Mikulincer, 2005). McClelland (1985) argued that conscious motivation is explicit and often focused on concrete, relatively immediate goals whereas unconscious motivation is implicit and is the primary driver of behavior over time. While explicit motivations do often override implicit motivations in the short term, unconscious motivations predicate the 
long-term behavior. Given that research also suggests a link between child-rearing practices and the power of implicit motivation (McClelland, 1985) and the link between these practices and culture, the psychodynamic perspective would predict significant differences across cultures in the dynamics of motivation.

The behaviorist perspective is not concerned specifically with constructs such as motivation but rather with operant conditioning. Operant conditioning means that people prefer to seek reward and avoid punishment. The theory focuses on drive-reduction, whereby the unfulfilled needs of humans lead to drives towards a state of homeostasis (Weiler, 2005). This drive towards a state of equilibrium is what motivates humans (Cheng \& Yeh, 2009). This perspective has a long history in education as witnessed by decades of research into schedules of reward for positive behavior and punishment for negative behavior. However, it has fallen out of favor in motivational theory in general as it applies to education in particular. This falling out of favor is due to the limitations of the perspective regarding explaining the wide diversity of human behavior (Cheng \& Yeh, 2009) and, in an educational context, its poor explanatory power for many types of learning (Weiler, 2005).

The humanistic perspective focuses on the need people have to grow and achieve a sense of personal identity and fulfillment. Maslow's hierarchy of needs is the dominant theory within this perspective. The theory argues that unfilled needs, organized in a hierarchy, drive motivation (Neher, 1991). The hierarchy progresses from lower-level needs to higher-level needs along the continuum: physiological, safety, love, and self-esteem. Each level of need must be satisfied to some extent before the next level becomes relevant. Thus, many people may feel stranded, and do not succeed in achieving their potential, forever driven and motivated by needs lower down in the hierarchy. The theory has spawned a vast research effort, particularly in industrial psychology, but many questions remain. Not only is the theory exceptionally difficult to test and validate, but researchers have been able to rigorously challenge its fundamental premises. Neher (1991), for example, challenged the notion that higher-order needs are innate, pointed out that in many societies the hierarchy did not work as described and argued that the theory, at best, only has limited application in affluent, western societies.

The cognitive perspective has come to dominate psychology in the last few decades, and it also leads research into motivation across many disciplines, not least of all education (Trautwein et al., 2012). The cognitive approach views the human mind as analogous to a computer and focuses on information processing as well as decision making (Trautwein et al., 2012). One of the dominant theories within this perspective is expectancy theory, which argues that perceived ability and perceived linkages between effort and achievement are central to motivation. Bandura (2000, 2004) points out that goals are at the core of cognitive, motivational theories, arguing that goals are outcomes desired by the individual that have their roots in social learning.

The goal-setting theory emerged from this focus on goals and had organizational contexts' research extensively focused on the theory (Locke, 1991). The emphasis here is on goals as outcomes that are desirable and deviate from the status quo. Locke (1991) argues that a specific set of conditions must exist before goals can motivate performance. In his argument, Locke mentioned that goals must be specific and relate to a gap between the current situation and the desired situation. He added that there must be a belief that the goal is attainable and regular feedback on progress is essential; goals must be at least moderately challenging, and the individual must be relatively committed to achievement. This theory is directly related to expectancy theory and has found significant support in educational contexts (Latham \& Brown, 2006).

In recent decades, an important branch of research within the cognitive perspective has focused on intrinsic versus extrinsic motivation. This research has challenged many of the key principles of the behaviorist approach and uncovered interesting parallels with the psychodynamic approach. Deci, Koestner and Ryan, (1999) challenged the focus of the behaviorists on rewards and punishments. They argued that rewards and punishments may act as extrinsic motivators and result in positive short-term outcomes, even to the extent of motivating people to develop competencies. Nevertheless, the most powerful motivators are those that are intrinsic, where people do things because they wish to do them. Deci et al. (1999) argue that rewards could, in fact, act as deterrents to the performance where people feel that rewards compromise their autonomy. They proposed self-determination theory which posits that motivation thrives in an environment that supports three innate needs- competence, autonomy, and relatedness. The correspondence to current thinking within the psychodynamic perspective is notable, with a direct link to relatedness and indirect links between self-esteem, competence and autonomy. New developments in self-determination theory 
further develop the link to the psychodynamic perspective with their focus on implicit motives, or motives that operate almost automatically at a level below that of consciousness.

\section{Motivation Unifying Themes}

Reeve, Bonaccio, and Winford (2014, p. 150) view motivation as a "force that energizes and directs people" where direction refers to purpose and goal-orientation while energy refers to "strength, intensity, and persistence." Reeve's (2008) point of departure is the unifying themes underlying the construct of motivation, as depicted in Figure 1.

Figure 1. Unifying Themes of Motivation

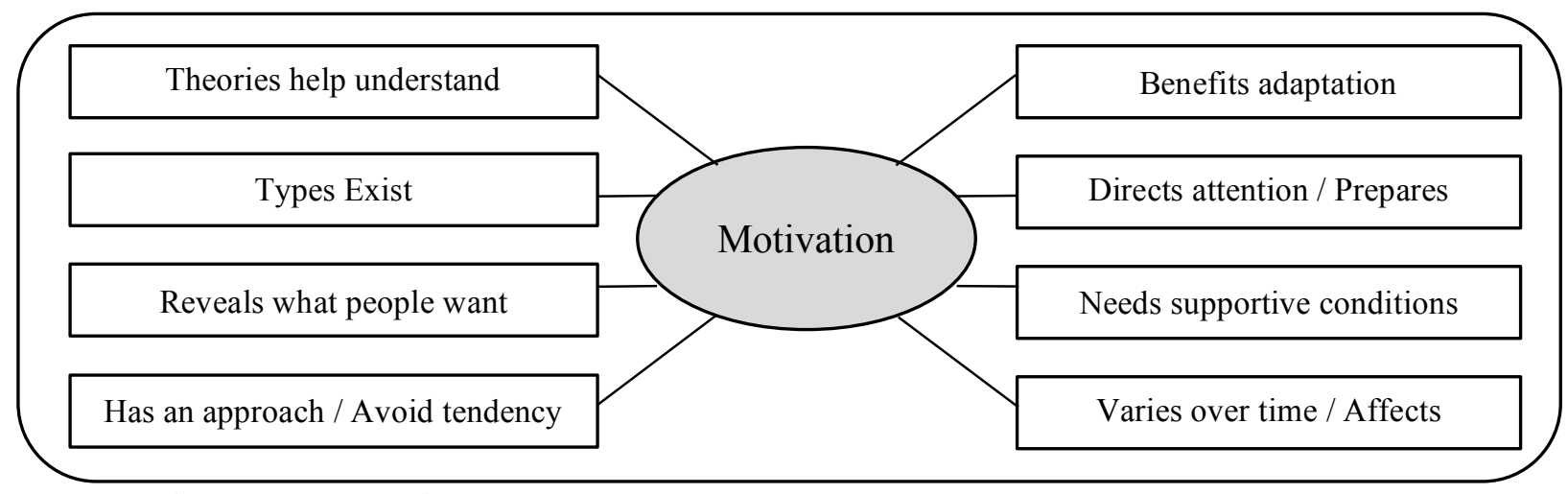

Source: (Reeve, 2008, pp. 13-14)

The first of the unifying assumptions is that motivation benefits adaptation (Reeve, 2008). Humans live in changing situations which offer opportunities and risks. In the face of constant environmental change, people need to preserve their well-being; they need to adapt. Motivation supplies humans with the resources that help them survive in such cases. The state of motivation in response to a particular situation can be both positive and negative, affecting the way people adapt. For example, students controlled by instructors, administrators, and parents might feel incapable of inner motivation and give up easily when faced with learning challenges. In contrast, giving students more autonomy and freedom of choice yields more will to initiate inner motivations, set goals, and show more persistence towards difficult learning tasks.

The second assumption is that "motives direct attention and prepare action"(Reeve, 2008, p. 14). For example, a student who is sitting in a dorm faced with three environmental events: friends giggling next door, feelings of fatigue, and a loud fire alarm. These three events trigger and give rise to three motives: affiliation that encourages the student to leave the room and ascertain why the friends are giggling; rest that demands the student gets some sleep; and the threat that demands the student to run down the stairs to avoid the danger. The level of urgency that will decide which motive will direct student action. In this case, because the threat motive has a higher precedence than the other motives, the student ends up exiting the dorm to avoid the threat associated with the sound of the fire alarm.

The third assumption is that motives change with time and impact our continuous behavior. People have, at any given time, a pool of motives and one can be dominant at some point in time while the others remain dormant, weakened but not gone, waiting for environmental triggers to re-energise them. Consider the former example of the student in the dorm: once the fire alarm is over and the associated conditions cease to exist, the threat motive is no longer dominant. The other two motives of affiliation with friends and sleep move up the hierarchy, and the drive to join friends to discuss what happened takes precedence over the rest motive.

The fourth assumption is that motivation is not a singular entity but a collection of different types. People are multifarious motivational creatures (Vallerand \& Losier, 1999). Consider two workers cutting stone. When asked what they are doing, one answers that he is cutting stone while the other mentions that he is building a castle. Two different types of motives lead to the same action, the motive to work, and the motive to achieve a goal. Both men are 
doing the same job, but the quality of their motivation differs. Thus, one is engaged in a routine daily task, and the other is more excited because of the more powerful motive he harbors. The former is a type of extrinsic motivation, where offering money to the worker yields the effort to cut stone, while the other is intrinsically motivated to fulfill an inner goal.

The fifth assumption is that motivation includes approach and avoidance inclinations. Humans adapt approach behavior when they want a desired outcome. On the other hand, they adapt avoidance behavior when they consider an undesired outcome. In the former example of the student in the dorm, the alarm sound directed the student to run away from an undesired outcome. Once that threat was over, the second motive of affiliation directed the student to approach friends to fulfill a desired outcome of being in a social group.

The sixth assumption is that the study of motivation reveals what people want. Motivation theories show and explain that there are similarities in people, even in different cultures and societies, in their emotions, biological dispositions, and needs. Nevertheless, these theories also point out that people grow their motivational assets through experience and via the surrounding cultural and societal framework. Thus, there are universal motivations, and others more specific, for example towards a particular culture or societal group. Thus, motivation theories when implemented associate which motivations are common to all humanity and which are more specific to particular environments.

The seventh assumption is that motivation depends on the environment for growth. If the environment is supportive, then motivation will flourish and lead to positive outcomes. On the other hand, when the environment is not supportive, negative actions dominate. For example, if students have more choice and empowerment over their learning, they will exhibit more positive actions and become more engaged in their studies. However, if students are controlled and not allowed freedom of choice, they adopt more negative reactions such as dropping out of college.

The eighth and last assumption is that motivation theories are essential to understanding the motivation. Motivation is an extremely complex construct, and there is a wealth of motivation theories that, when utilized correctly, can help researchers understand an issue and then guide the solution to it. Without these theoretical frameworks, the inner workings of motivation would be unfathomable.

Reeve (2008) argues that the sources of motivation include needs, cognitions, and emotions, as shown in Figure 2.

Figure 2. Sources of Motivation

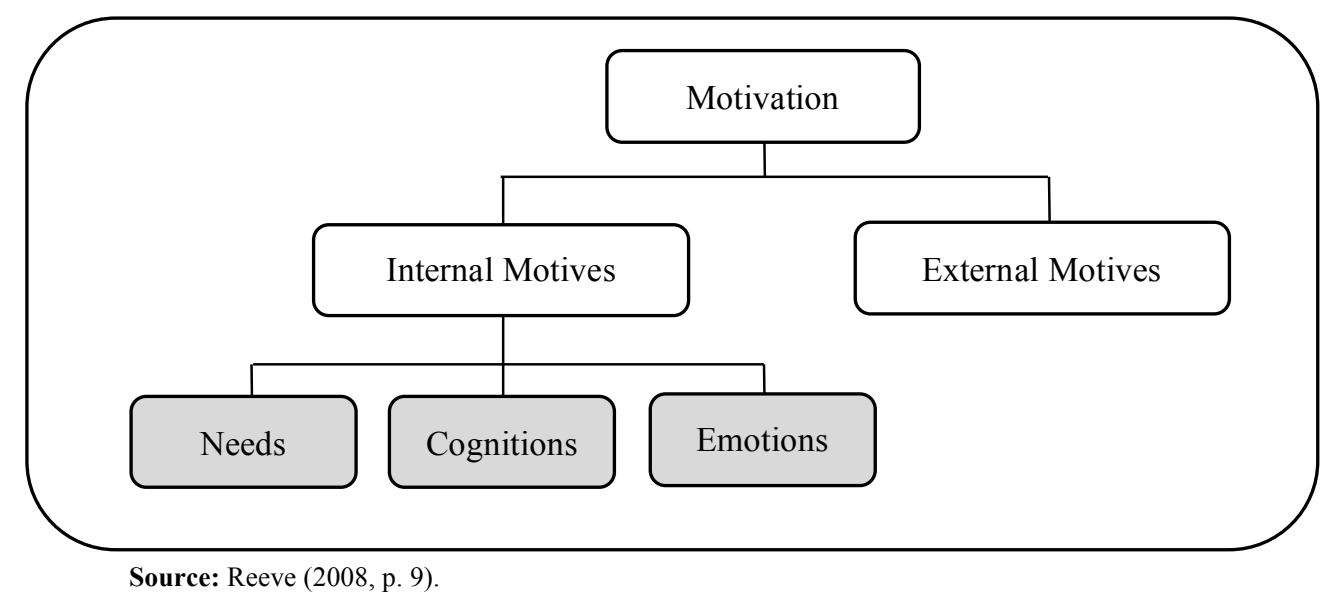

Figure 2 highlights the interplay of internal motivational forces and the external environment. The external events might include characteristics of the task environment, situational constraints, and sociocultural factors. Motivation is a "private, unobservable, and seemingly mysterious experience" (Reeve, 2008, p. 10) and as a result, researchers cannot observe motivation directly. Instead, research can observe the behaviors that motivate or guide certain actions. Direct observation of behavior in controlled environments or via expressions of motivation such as observable 
behavior, engagement, physiological changes and self-reports enables indirect motivation measurement (Reeve, 2008).

Learning about motivation through observation is similar to lab experiments which scientists conduct in a controlled environment and register the outcome. Given the difficulties associated with this approach, the alternative approach of observing expressions of motivation dominates the research. There are four expressions of motivation: behavior, engagement, physiology and self-report, as depicted inTable 1 below.

Table 1. Expressions of Motivation

\begin{tabular}{|c|c|c|}
\hline Motivation Expression Type & Behavior & Engagement \\
\hline $\begin{array}{l}\text { Motivation Expression } \\
\text { Elements }\end{array}$ & $\begin{array}{l}\text { - } \text { Probability of response } \\
\text { - } \text { Facial Expressions } \\
\text { - } \text { Bodily Gesture } \\
\text { - Attention } \\
\text { - Effort } \\
\text { - Persistence } \\
\text { - Choice }\end{array}$ & $\begin{array}{l}\text { Behavioral Engagement (attention, effort, } \\
\text { persistence) } \\
\text { Emotional engagement (interest, enjoyment, sadness, } \\
\text { anger) } \\
\text { - Cognitive engagement (deep processing, Strategies } \\
\text { (learning strategy, problem-solving strategy)), Self- } \\
\text { Regulation. } \\
\text { - Voice Self-expressions (expressions of needs, } \\
\text { preferences, \& desires of the self), participation. }\end{array}$ \\
\hline Motivation Expression Type & Physiology & Self- Report \\
\hline $\begin{array}{l}\text { Motivation Expression } \\
\text { Elements }\end{array}$ & - Physiological reactions & - $\mathrm{Q} \& \mathrm{~A}$ \\
\hline
\end{tabular}

Source: (Reeve, 2008)

The first expression of motivation is behavior. Eight elements in behavior represent presence, intensity, and quality of motivation. These are attention, effort, latency, persistence, choice, the probability of response, facial expressions and bodily gestures. The presence or lack of these elements indicates weak or intense motives within a person.

The second expression of motivation is engagement. The term engagement refers to "a student's active involvement in a learning activity" (Reeve, 2012, p. 150). Engagement is a multidimensional construct comprising four highly inter-correlated dimensions, as follows:

- Behavioral engagement relates to the more visible aspects of student engagement, including effort, participation, tenacity and compliance with rules (Reeve, 2008). The eight elements of behavioral engagement are attention, effort, latency, persistence, choice, the probability of response, facial expressions and bodily gestures. The presence or lack of these elements indicates weak or intense motives within a person. While the engaged student may be hard-working and compliant, this does not ensure that learning is taking place.

- Cognitive engagement is required before true learning takes place (Reeve, 2008). Essentially, behavioral engagement refers to the quantity of the engagement while cognitive engagement is a more qualitative concept. The cognitively engaged student understands and masters the material while managing the learning process (Reeve, 2008). The focus here is on the intensity and quality of the engagement behavior, emotion, and cognitions. This focus also includes the monitoring of the self's expressions of its needs.

- Emotional engagement relates to feelings of, for example, happiness, frustration, joy and anger about the institution, curriculum, and fellow students. The emotionally engaged student is positive, enthusiastic and feels part of the institution (Reeve, 2008). Some theorists view emotional engagement as a form of relational engagement involving the extent to which the student feels there are personal attachment and relationship with the institution, teachers and fellow students (Reeve, 2008).

- Voice relates to actual participation where the individual expresses opinions, desires, preferences, needs and so on. 
The third expression of motivation is physiological reactions. Here, physiological responses are the focus, for example, changes in the brain, hormones, heart rate, blood chemistry, and respiratory system. Physiological motivation is beyond the scope of this study.

The last expression of motivation is self-report. In self-report, people talk about their motivation in a participatory fashion when asked. Asking questions, whether survey or open-ended questions, is a quick and effective way to retrieve specific behavioral expressions of motivation. Using this method of motivational inquiry means that the obtained data is reliant on how accurately people describe their behavior. Therefore, it is important that participants trust the interviewer and feel comfortable and safe sharing their emotions and behavioral experiences.

\section{Student Motivation Research}

Motivation is a complex entity that has spawned many theories and models (Chaudhary, 2014; Rizwan, Tariq, Hassan, \& Sultan, 2014). It is essential to study the cause of the human behavior and the changes it goes through to understand the associated motivation (Nuttin, 2014). The sheer breadth of the research in this area is so vast that focus is critical in any research project. In the context of student motivation research, student motivation relates to multiple theorybased constructs such as interests, goals, self-efficacy and attributes that shape student motivation and impact academic outcomes (Pintrich, 2000). Buehl and Alexander (2009) point out the importance of "self-efficacy, competency beliefs, task value and interest, self-determination and goal orientation" (p. 479) in the study of motivation. The cognitive perspective is best suited to motivation research regarding its relative theoretical adequacy.

The aim of research on student motivation is to explore direct and indirect influences on student motivation and their impact on student experience. The first line of inquiry in understanding motivation is to comprehend how specific behaviors evolve. Motivation impacts behavior initiation, direction, strength, and cessation. For example, why do people begin an activity? Why do they continue this activity? Why did they choose this activity over others? Why did they stop the activity? And will they repeat such activity again? Thus, the question of what causes behavior may appear to be somewhat simplistic but is in fact highly complex. It includes all those elements necessary to understand the role of motivation in nurturing, impacting and explaining ongoing behavior.

The second line of inquiry in the quest to understand motivation is to understand fluctuations in behavioral intensity. This insight is essential to the knowledge of the different reactions of behavioral activism and passivism within a person on different occasions or between people reacting to the same environmental event. Motivation shapes behavior (Bahlmann, Aarts, \& D'Esposito, 2015), changes over time (Kim, 2015) and waxes and wanes at times (Benedetti, Diefendorff, Gabriel, \& Chandler, 2015)

\section{A Student Motivation Research Framework}

As a whole, motivation theory offers an understanding of how behavior forms and changes over time, also accounting for individual differences. Motivation is a process that forms, energizes, and directs behavior. In essence, there are internal processes in the inner self, and external processes arise from the surrounding environment that initiates human behavior. Even when narrowing the theoretical scope of the research down to the cognitive perspective combined with some elements of the psychodynamic perspective, it soon becomes clear that the diversity of approaches and theories requires a conceptual model to shape and structure the research effort.

Reeve (2008) proposed a conceptual framework for motivation that aligns well with student motivation research. His framework emanates from self-determination theory which focuses on the interaction of inner motivational resources, innate growth characteristics, needs and sociocultural factors as people strive to develop and achieve a state of autonomous self-regulation (Deci \& Ryan, 2012c).

The intent of the motivation framework is to connect expressions of motivation to their initiating events and underlying motivational theories. Reeves' (2008) framework, as shown in Figure 3 below, offers the mechanism to express such relationships. Here Reeve lays out the dynamics of the motivational process and the various forces that are in operation. 
Figure 3. Motivation Framework



In the model, applying motivation theories to triggering conditions of motivation represents a systems flow leading to specific outcomes. As seen before, motivation theories are practical when they predict antecedent conditions' outcomes accurately. Conditions in the environment interact with internal states to create a sense of urgency to act. The motive status is where motivation theories of needs, cognitions and emotions work to predict the energy and direction of one's desire to approach or avoid, want to or not want, which leads to one of the four expressions of motivation. For educational field research, measurement issues imply the discount of physiology as an energizing and directing method.

The derived model shown in Figure 4 drives data collection. The model hypothesizes that students are more likely to talk about what they faced that directed their behavior and impacted their motivations and learning. More specifically, students' self-reports will provide insights into the antecedent conditions they face, the dynamic interplay of needs, cognitions and emotions that drive them to respond in certain ways and, of greatest importance, their unique responses. 
Figure 4. A Student Motivation Research Framework

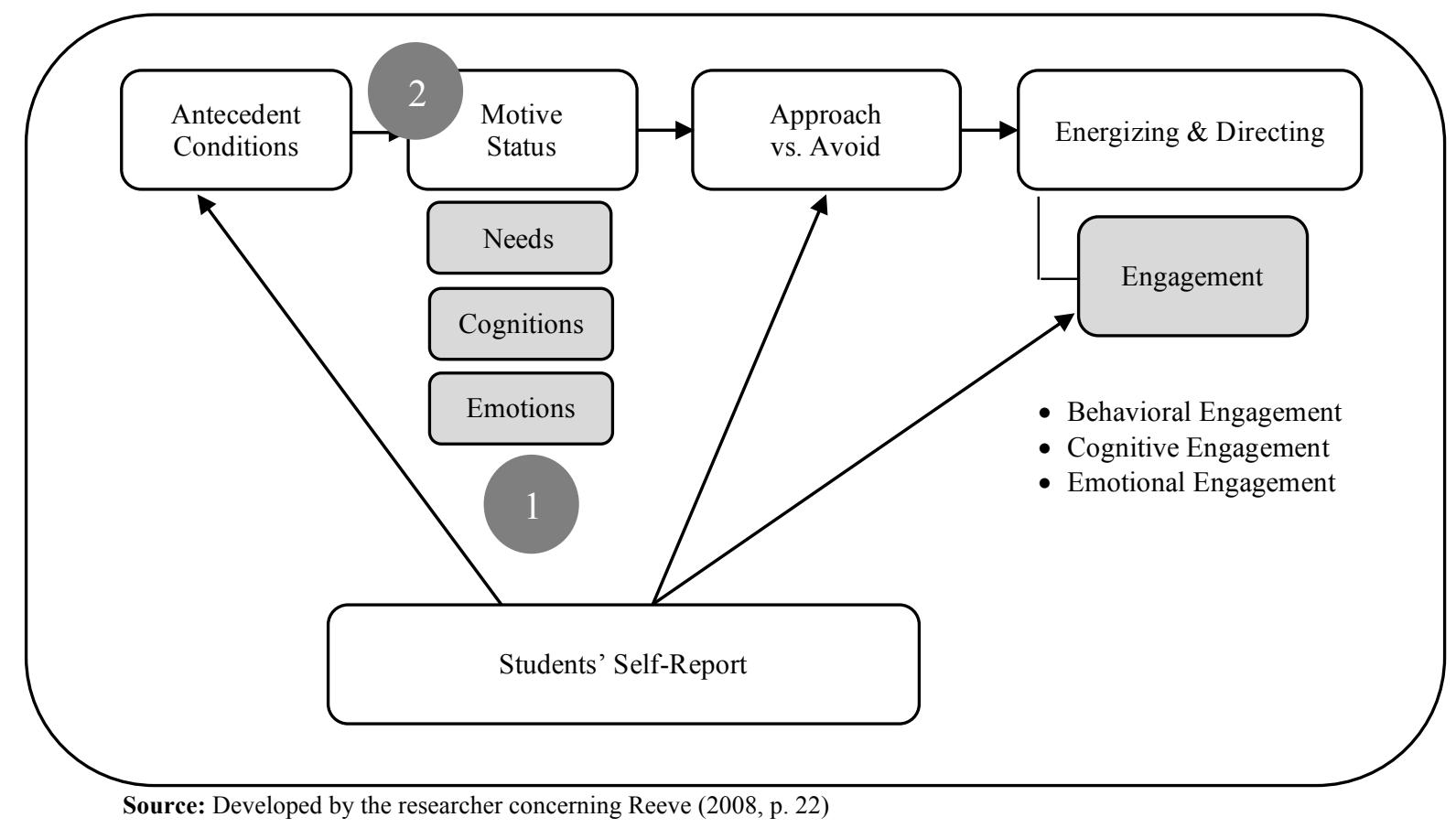

The conceptual framework focuses on cognition, needs, and emotion (affect). This framework aligns well with the theoretical impetus towards cognitive explanations without discounting the influence of needs, which have their roots in psychodynamic theory. The framework also acknowledges the influence of emotion on motivation. The framework proposes that the interplay of antecedent conditions and internal motives will drive approach or avoidance behavior which in turn results in engagement (or otherwise) at the behavioral, cognitive and emotional levels. There are now a significant number of theories of motivation, and it is not possible to address all of them in a single research effort. Given the focus on the interplay of antecedent conditions and internal motivational states, we recommend the use of some theories of motivation that provide a theoretical background and align with the suggested framework in conducting research on student motivation.

\section{ACKNOWLEDGEMENT}

A portion of this article is taken from the main author's $\mathrm{PhD}$ thesis (Alkaabi, 2016).

\section{REFERENCES}

Alkaabi, S. (2016) Determinants that impact first year male students' motivation to learn at UAE public colleges USQ ePrints https://eprints.usq.edu.au/28742/

Bahlmann, J., Aarts, E., \& D'Esposito, M. (2015). Influence of motivation on control hierarchy in the human frontal cortex. The Journal of Neuroscience, 35(7), 3207-3217.

Bandura, A. (2000). Self-efficacy: The foundation of agency1. In w. Perrig, J. \& A. Grob (Eds.), Control of human behavior, mental processes, and consciousness (pp. 16-30). Universitat Bern, Switzerland: Psychology Press.

Bandura, A. (2004). Health promotion by social cognitive means. Health Education \& Behavior, 31(2), $143-164$.

Benedetti, A. A., Diefendorff, J. M., Gabriel, A. S., \& Chandler, M. M. (2015). The effects of intrinsic and extrinsic sources of motivation on well-being depend on time of day: The moderating effects of workday accumulation. Journal of Vocational Behavior, 88, 38-46.

Buehl, M. M., \& Alexander, P. A. (2009). Beliefs about learning in academic domains. In W. K. R. \& A. Wigfield (Eds.), Handbook of Motivation at School (pp. 479-501). New York: Routledge.

Chaudhary, N. (2014). Role of motivation in talent retention and increasing productivity. Suresh Gyan Vihar University. Retrieved from http://hdl.handle.net/10603/25024

Cheng, Y. C., \& Yeh, H. T. (2009). From concepts of motivation to its application in instructional design: Reconsidering 
motivation from an instructional design perspective. British Journal of Educational Technology, 40(4), 597-605.

Deci, E. L., Koestner, R., \& Ryan, R. M. (1999). A meta-analytic review of experiments examining the effects of extrinsic rewards on intrinsic motivation. Psychological bulletin, 125(6), 627.

Deci, E. L., \& Ryan, R. M. (2012). Motivation, personality, and development within embedded social context: An overview of self-determination theory. In R. Richard M. (Ed.), Th Oxford Handbook of Human Motivation (pp. 85-110). NY: Oxford University Press, Inc.

Jenkins, L. N., \& Demaray, M. K. (2015). An investigation of relations among academic enablers and reading outcomes. Psychology in the Schools, 52(4), 379-389.

Kim, K. J. (2015). Motivation and its changes of Korean EFL middle school students. Retrieved from: http://210.101.116.28/kiss9/preview.asp

Latham, G. P., \& Brown, T. C. (2006). The effect of learning vs. outcome goals on self-Efficacy, satisfaction and performance in an MBA program. Applied Psychology, 55(4), 606-623.

Locke, E. A. (1991). Goal theory vs. control theory: Contrasting approaches to understanding work motivation. Motivation and Emotion, 15(1), 9-28.

McClelland, D. C. (1985). How motives, skills, and values determine what people do. American psychologist, $40(7), 812$.

Mitchell, S. A. (1988). Relational concepts in psychoanalysis: Harvard University Press.

Neher, A. (1991). Maslow's Theory of Motivation A Critique. Journal of Humanistic Psychology, 31(3), 89-112.

Nuttin, J. (2014). Future time perspective and motivation: Theory and research method. NY: Psychology Press.

Pintrich, P. R. (2000). An achievement goal theory perspective on issues in motivation terminology, theory, and research. Contemporary Educational Psychology, 25(1), 92-104.

Reeve, C. L., Bonaccio, S., \& Winford, E. C. (2014). Cognitive ability, exam-related emotions and exam performance: A field study in a college setting. Contemporary Educational Psychology, 39(2), 124-133.

Reeve, J. (2008). Understanding Motivation and Emotion (5th ed.). London: John Wiley \& Sons, Inc.

Reeve, J. (2012). A self-determination theory perspective on student engagement. In Handbook of research on student engagement (pp. 149-172). Springer US.

Rizwan, M., Tariq, M., Hassan, R., \& Sultan, A. (2014). A comparative analysis of the factors effecting the employee motivation and employee performance in Pakistan. International Journal of Human Resource Studies, 4(3), 35-49.

Ryan, R. M. (2012). Motivation and the organization of human behavior: Three reasons for the reemergence of a field. In R. M. Ryan (Ed.), The Oxford handbook of human motivation (pp. 3-9). U.S: Oxford University Press.

Schunk, D. H., Pintrich, P. R., \& Meece, J. L. (2008). Motivation in education. Upper Saddle River, NJ: Person Education.

Shaver, P. R., \& Mikulincer, M. (2005). Attachment theory and research: Resurrection of the psychodynamic approach to personality. Journal of Research in Personality, 39(1), 22-45.

Trautwein, U., Marsh, H. W., Nagengast, B., Lüdtke, O., Nagy, G., \& Jonkmann, K. (2012). Probing for the multiplicative term in modern expectancy-value theory: A latent interaction modeling study. Journal of Educational Psychology, 104(3), 763.

Vallerand, R. J., \& Losier, G. F. (1999). An integrative analysis of intrinsic and extrinsic motivation in sport. Journal of Applied Sport Psychology, 11(1), 142-169.

Weiler, A. (2005). Information-seeking behavior in Generation Y students: Motivation, critical thinking, and learning theory. The Journal of Academic Librarianship, 31(1), 46-53.

Weiner, B. (1992). Human motivation: Metaphors, theories, and research. Newbury Park, CA.: Sage.

Wigfield, A., Eccles, J. S., Schiefele, U., Roeser, R. W., \& Davis-Kean, P. (2009). Development of achievement motivation. In W. Damon (Ed.), Handbook of child psychology: Vol. 3. Social, emotional, and personality development (Vol. 3, pp. 933-1002). Hoboken, NJ: Wiley. 
NOTES 